\title{
Improving the Pediatric Hospital Experience Using Telepresence Robotics
}

\author{
Galen Shi ${ }^{1 *}$, BA; Pavan Shah ${ }^{1 *}$, BS; Therese Canares ${ }^{2}$, MD; Ingrid Zimmer-Galler ${ }^{3}$, MD \\ ${ }_{1}^{1}$ Johns Hopkins University School of Medicine, Baltimore, MD, United States \\ ${ }^{2}$ Pediatrics - Emergency Medicine, Johns Hopkins University School of Medicine, Baltimore, MD, United States \\ ${ }^{3}$ Johns Hopkins Wilmer Eye Institute, Johns Hopkins University School of Medicine, Baltimore, MD, United States \\ *these authors contributed equally
}

\section{Corresponding Author:}

Galen Shi, BA

Johns Hopkins University School of Medicine

733 N Broadway

Baltimore, MD

United States

Phone: 4438968584

Email: gshi8@jhmi.edu

\begin{abstract}
Background: In a hospital setting, pediatric patients miss out on routine stress coping mechanisms, such as normative play, socialization, and exploration. Previous work has established that a lack of healthy coping mechanisms negatively impacts the psychosocial development and well-being of hospitalized children. To help alleviate the stress of hospitalization, we created a novel, normative play robotics program that utilizes telepresence technology to give children live, virtual tours of museums and cultural attractions.
\end{abstract}

Objective: The objective was to 1) demonstrate feasibility, and 2) assess quality and impact on mood of telepresence tours as a new normative play intervention.

Methods: A prospective, cross-sectional study was conducted on inpatients at the Johns Hopkins Children's Center and the University of Maryland Children's Hospital from the ages of 3 to 19. Subjects were selected through convenience sampling by Child Life Specialists. The intervention consisted of a 30-minute session in which a volunteer: 1) remotely connected a laptop in the hospital room to a telepresence robot at the Maryland Science Center or the National Aquarium; 2) introduced the child to their venue tour guide and taught the child how to drive the robot, and 3) facilitated a tour in conjunction with the venue tour guide on site with the robot. Feasibility was assessed by the rate of completed tours. Quality of the experience and mood were evaluated with surveys answered with a 5 or 7-point Likert scale. Surveys were administered in 2 phases: phase 1 evaluated quality of the experience after the tour, and phase 2 included the phase 1 survey plus mood assessment before and after the tour. The two-tailed Wilcoxon signed-rank test was used to determine statistical significance in difference in mood before and after the tour. Verbal consent was obtained from patient guardians and assent was obtained from the patient.

Results: 77 tours were conducted with pediatric inpatients, and no tours were terminated due to technical difficulty. Out of 77 children, 68 completed surveys. In phase $1(n=40)$ participants' average age was 7.8 years. On average, patients rated their overall experience a $4.8 / 5$, ease of driving $4.1 / 5$, ease of communicating with other guests at the venues a $4.3 / 5$, and their tour guides a $4.7 / 5$. In phase $2(n=37)$, average age was 10.1 years. Children's self-reported mood improved from pre-visit (3.7/5) to post visit $(4.4 / 5)(P<.001)$. Additionally, patients rated their overall ease of driving 5.8/7, ease of communicating with other guests at the venues 5.3/7, and their tour guides 6.8/7.

Conclusions: Patients reported positive assessments of their overall experience, ease of driving, communication with tour guides and guests at the venues, and elevated mood after the tour. This study demonstrates that telepresence tours are a feasible and enjoyable intervention for hospitalized pediatric patients, and significantly improve mood. This unique program adds to existing child life specialist strategies by allowing children to engage in normative behaviors outside of the hospital. This novel use of telepresence robotics as a normative play intervention warrants future studies to further characterize the improvement in mood and impact on the patient's overall hospital satisfaction.

(iproc 2019;5(1):e15259) doi: 10.2196/15259 


\section{KEYWORDS}

affect; asessment; children and adolescents; mood; survey; telemedicine

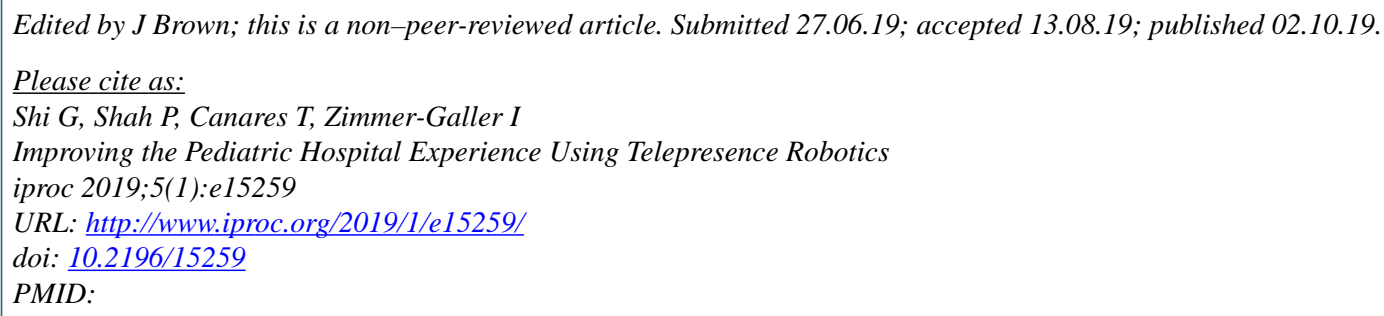

(C)Galen Shi, Pavan Shah, Therese Canares, Ingrid Zimmer-Galler. Originally published in Iproceedings (http://www.iproc.org), 02.10.2019 This is an open-access article distributed under the terms of the Creative Commons Attribution License (https://creativecommons.org/licenses/by/4.0/), which permits unrestricted use, distribution, and reproduction in any medium, provided the original work, first published in Iproceedings, is properly cited. The complete bibliographic information, a link to the original publication on http://www.iproc.org/, as well as this copyright and license information must be included. 